\title{
Online Appendix: A Central Bank Theory of Price Level Determination
}

\author{
Pierpaolo Benigno \\ LUISS and EIEF
}

July 1, 2019

\author{
Abstract \\ This Appendix presents the case in which long-term securities are \\ held by the central bank.
}




\section{Unconventional open-market operations}

In this Appendix, I extend the model of Section 2 of the main text to allow for long-term securities in order to analyze how results change when the central bank engages in unconventional open-market operations and holds risky securities in its balance sheet.

\subsection{Consumers}

Consumers have preferences:

$$
\sum_{t=t_{0}}^{\infty} \beta^{t-t_{0}} u\left(c_{t}\right)
$$

where $\beta$ is the intertemporal discount factor with $0<\beta<1, c$ is a consumption good and $u(\cdot)$ is a concave function, twice continuously differentiable, increasing in $c$.

The consumers' budget constraint is:

$$
\frac{B_{t}+X_{t}}{1+i_{t}}+Q_{t} D_{t} \leq B_{t-1}+X_{t-1}+\left(1-\varkappa_{t}\right)\left(1+\delta Q_{t}\right) D_{t-1}+P_{t}\left(y-c_{t}\right)-T_{t}^{F} .
$$

Consumers can invest their financial wealth in interest-bearing reserves, $X_{t}$, issued by the central bank at the risk-free nominal interest rate $i_{t}$ and can lend or borrow using short-term securities, $B_{t}$, at the same interest rate $i_{t}$. $D_{t}$ indicates holdings of long-term securities issued at a price $Q_{t}$. The security available has decaying coupons: by lending $Q_{t}$ units of currency at time $t$, geometrically decaying coupons are delivered equal to $1, \delta, \delta^{2}, \delta^{3} \ldots$ in the following periods and in the case of no default. ${ }^{1}$ The variable $\varkappa_{t}$ on the right-hand side of (2) captures the possibility that long-term securities can be partially seized by exogenous default. $y$ is a constant endowment of the only good traded; $T_{t}^{F}$ are lump-sum taxes levied by the treasury. There are no financial markets before time $t_{0}$, therefore $B_{t_{0}-1}, X_{t_{0}-1}, D_{t_{0}-1}$ are all equal to zero.

The consumers' problem is subject to a borrowing limit of the form

$$
\lim _{T \rightarrow \infty}\left\{R_{t_{0}, T}\left(\frac{B_{T}+X_{T}}{1+i_{T}}+Q_{T} D_{T}\right)\right\} \geq 0
$$

\footnotetext{
${ }^{1}$ The stock of long-term asset follows the law of motion $D_{t}=Z_{t}+(1-\delta) D_{t-1}$, where $Z_{t}$ is the amount of new long-term lending, if positive, supplied at time $t$. See among others Woodford (2001).
} 
and to the bound

$$
\sum_{T=t_{0}}^{\infty} R_{t_{0}, T} P_{T} c_{T}<\infty
$$

since there is no limit to the ability of households to borrow against future income.

Households choose consumption, and asset allocations to maximize utility (1) under constraints (2), (3), (4) given the initial conditions. The set of firstorder conditions imply the Euler equation

$$
\frac{u_{c}\left(c_{t}\right)}{P_{t}}=\beta\left(1+i_{t}\right) \frac{u_{c}\left(c_{t+1}\right)}{P_{t+1}}
$$

at each time $t \geq t_{0}$ assuming interior solution.

Absence of arbitrage opportunities implies that

$$
Q_{t}=\beta \frac{u_{c}\left(c_{t+1}\right)}{u_{c}\left(c_{t}\right)} \frac{P_{t}}{P_{t+1}}\left(1-\varkappa_{t+1}\right)\left(1+\delta Q_{t+1}\right)
$$

from which a "fundamental" solution for long-term bond prices follows:

$$
Q_{t}=\sum_{T=t}^{\infty} \delta^{T-t} \beta^{T+1-t} \frac{u_{c}\left(c_{T+1}\right)}{u_{c}\left(c_{t}\right)}\left(\frac{P_{t}}{P_{T+1}}\right) \prod_{j=t+1}^{T+1}\left(1-\varkappa_{j}\right)
$$

at each time $t \geq t_{0}$.

In a perfect-foresight equilibrium the return on long-term bonds is also equal to the short-term interest rate as shown by combining (5) and (6)

$$
r_{t+1}=i_{t}
$$

with the return on long-term bonds defined by $r_{t+1} \equiv\left(1-\varkappa_{t+1}\right)\left(1+\delta Q_{t+1}\right) / Q_{t}-$ 1 .

To conclude the characterization of the consumer's problem, a transversality condition applies and therefore (3) holds with equality, given the equilibrium nominal stochastic discount factor

$$
R_{t_{0}, T}=\beta^{T-t_{0}} \frac{u_{c}\left(c_{T}\right)}{u_{c}\left(c_{t_{0}}\right)} \frac{P_{t_{0}}}{P_{T}}
$$




\subsection{Treasury}

The treasury raises lump-sum taxes $T_{t}^{F}$ (net of transfers) from the private sector and receives remittances $T^{C}$ (when $T^{C}$ is positive) or makes transfers to the central bank (when $T^{C}$ is negative). The treasury can finance its deficit through short-term debt $\left(B^{F}\right)$ at the price $1 /\left(1+i_{t}\right)$, facing the following flow budget constraint

$$
\frac{B_{t}^{F}}{1+i_{t}}=B_{t-1}^{F}-T_{t}^{F}-T_{t}^{C}
$$

given initial condition $B_{t_{0}-1}^{F}=0$. To simplify the analysis, I assume that the treasury does not issue long-term securities.

\subsection{Central Bank}

The central bank can invest in short and long-term securities, $B_{t}^{C}$ and $D_{t}^{C}$, by issuing reserves $X_{t}^{C}$. Net worth, $N_{t}^{C}$ is defined as

$$
N_{t}^{C} \equiv Q_{t} D_{t}^{C}+\frac{B_{t}^{C}}{1+i_{t}}-\frac{X_{t}^{C}}{1+i_{t}}
$$

with law of motion given by:

$$
N_{t}^{C}=N_{t-1}^{C}+\Psi_{t}^{C}-T_{t}^{C}
$$

where $\Psi_{t}^{C}$ are central bank's profits:

$$
\Psi_{t}^{C}=i_{t-1} N_{t-1}^{C}+\left(r_{t}-i_{t-1}\right) Q_{t-1} D_{t-1}^{C} .
$$

Combining (8), (9) and (10), the central bank's flow budget constraint follows:

$$
Q_{t} D_{t}^{C}+\frac{B_{t}^{C}}{1+i_{t}}-\frac{X_{t}^{C}}{1+i_{t}}=\left(1-\varkappa_{t}\right)\left(1+\delta Q_{t}\right) D_{t-1}^{C}+B_{t-1}^{C}-X_{t-1}^{C}-T_{t}^{C},
$$

given initial conditions $D_{t_{0}-1}^{C}, B_{t_{0}-1}^{C}, X_{t_{0}-1}^{C}$, all equal to zero.

\subsection{Equilibrium}

Equilibrium in the goods market implies that

$$
c_{t}=y,
$$


at each time $t \geq t_{0}$ while equilibrium in the asset markets that

$$
\begin{gathered}
B_{t}+B_{t}^{C}=B_{t}^{F}, \\
X_{t}=X_{t}^{C}, \\
D_{t}+D_{t}^{C}=0 .
\end{gathered}
$$

\subsection{Equilibrium conditions}

I now characterize in a compact way the equilibrium conditions of the model.

The Fisher's equation follows from the Euler equation (5) using equilibrium in the goods market

$$
1+i_{t}=\frac{1}{\beta} \frac{P_{t+1}}{P_{t}}
$$

while the equilibrium price of long-term securities is:

$$
Q_{t}=\sum_{T=t}^{\infty} \delta^{T-t} \beta^{T+1-t}\left(\frac{P_{t}}{P_{T+1}}\right) \prod_{j=t+1}^{T+1}\left(1-\varkappa_{j}\right) .
$$

The household's transversality condition can be simplified to

$$
\lim _{T \rightarrow \infty}\left\{\beta^{T-t_{0}}\left(\frac{P_{t_{0}}}{P_{T}}\right)\left(\frac{B_{T}+X_{T}}{1+i_{T}}+Q_{T} D_{T}\right)\right\}=0
$$

while the bound (4) can be written as

$$
\sum_{T=t_{0}}^{\infty} \beta^{T-t_{0}} y<\infty
$$

which is naturally satisfied.

The flow budget constraints of treasury and central bank are respectively

$$
\begin{gathered}
\frac{B_{t}^{F}}{1+i_{t}}=B_{t-1}^{F}-T_{t}^{F}-T_{t}^{C} \\
Q_{t} D_{t}^{C}+\frac{B_{t}^{C}}{1+i_{t}}-\frac{X_{t}^{C}}{1+i_{t}}=\left(1-\varkappa_{t}\right)\left(1+\delta Q_{t}\right) D_{t-1}^{C}+B_{t-1}^{C}-X_{t-1}^{C}-T_{t}^{C},
\end{gathered}
$$


while equilibrium in the securities market closes the model

$$
\begin{gathered}
B_{t}+B_{t}^{C}=B_{t}^{F}, \\
X_{t}=X_{t}^{C}, \\
D_{t}+D_{t}^{C}=0 .
\end{gathered}
$$

A rational-expectations equilibrium is a collection of processes $\left\{P_{t}, i_{t}, Q_{t}\right.$, $\left.T_{t}^{F}, T_{t}^{C}, B_{t}, B_{t}^{C}, B_{t}^{F}, D_{t}^{C}, X_{t}\right\}_{t=t_{0}}^{\infty}$ that satisfy (11)-(16) at each date $t \geq t_{0}$ given (17)-(18) and initial conditions $B_{t_{0}-1}^{C}, B_{t_{0}-1}^{F}, D_{t_{0}-1}, X_{t_{0}-1}$ all equal to zero. Since (13) is a bound, there are five degrees of freedom to specify the monetary/fiscal policy regime. Note equation (13) can be replaced by (??) which is an equivalent condition using equations (14)-(18).

I can now define equilibrium in this more general model.

Definition 1 An equilibrium is a set of sequences $\left\{P_{t}, i_{t}, T_{t}, T_{t}^{F}, T_{t}^{C}, B_{t}^{F}\right.$, $\left.B_{t}^{C}, D_{t}^{C}, X_{t}^{C}\right\}_{t=t_{0}}^{\infty}$ with $P_{t}, i_{t} \geq 0$ that solve equations (??), (??), (??),

$T_{t}=T_{t}^{F}$, and (15), given the specification of the monetary/fiscal policy regime which sets the sequences $\left\{i_{t}, T_{t}^{F}, T_{t}^{C}, X_{t}, D_{t}^{C}\right\}_{t=t_{0}}^{\infty}$, given initial conditions $B_{t_{0}-1}^{F}=B_{t_{0}-1}^{C}=D_{t_{0}-1}^{C}=X_{t_{0}-1}^{C}=0$ and the definition $\tau_{t}=T_{t} / P_{t}$.

There are now four restrictions for the nine unknowns leaving five degrees of freedom to specify the monetary/fiscal policy regime. On top of the specifications given in Definitions ?? and ??, I need to set the sequence $\left\{D_{t}^{C}\right\}_{t=t_{0}}^{\infty}$ of long-term asset purchases by the central bank.

\subsection{Results with treasury's support}

By holding long-term bonds, the central bank can be subject to income losses due to unforeseen shocks. However, the results of Section ?? on how to trim deflationary spirals hold even in this more general framework, as shown in the following Proposition.

Proposition 2 Given the monetary/fiscal policy regime of Definition ?? and a non-negative sequence $\left\{D_{t}^{C}\right\}_{t=t_{0}}^{\infty}$, it follows that $P_{t} \geq P^{*}$ in equilibrium for each $t \geq t_{0}$. 
Proof. As in Proposition ??.

The key element for extending the result of Section ?? is to interpret the transfer rule $T_{t}^{C}=\Psi_{t}^{C}$ in a symmetric way. In particular, the rule implies that the treasury is committed to transfer resources to the central bank in the case of negative profits. $^{2}$ Given the initial capitalization and the commitment to $T_{t}^{C}=\Psi_{t}^{C}$, central bank's nominal net worth remains constant and all the discussion of Section ?? to eliminate deflationary spirals applies to this more general context.

However, in this case, the central bank is no longer financially independent from the treasury which brings about the risk that it could be asked to remit additional dividends at the treasury's will. What is going to be weakened, in this case, is the strength of the commitment that rules out deflationary paths, as discussed in Section ??. If this weakness is understood by the private sector then deflations can develop unraveling the uniqueness of equilibrium.

The result of this section can be consistent with the story of a central bank that undertakes unconventional open-market operations with a deflation going on. In this environment, it is also possible that the central bank derives profits from its holdings of risky assets, as a consequence of unexpected deflationary shocks, and can therefore rebate income to the treasury. However, it is understood that in the case of losses - following perhaps a future exit from a policy of zero nominal interest rates - the treasury stands ready to support the central bank. This implicit support might be enough to undermine the financial independence of the central bank during the zero interest-rate policy because it suggests that it could be in the treasury's ability to expropriate central bank's net worth. Although these raids are not in the observation period, the expectation that they will occur is sufficient to validate the deflationary path.

The results of Section ?? to eliminate inflationary spirals extend as well to the case in which the central bank holds long-term securities.

Proposition 3 Given the monetary/fiscal policy regime of Definition ?? and a non-negative sequence $\left\{D_{t}^{C}\right\}_{t=t_{0}}^{\infty}$, it follows that $P_{t}=P^{*}$ in equilibrium for each $t \geq t_{0}$.

\footnotetext{
${ }^{2}$ An interesting example in the recent financial crisis of explicit treasury's support is that of the Bank of England which in January 2009 established a wholly-owned subsidiary with the responsibility of buying private and public long-term securities. The company is fully indemnified by the Treasury since any financial losses are borne by the Treasury and any gains are owed to the Treasury.
} 
Proof. As in Proposition ??.

Even in this case, nothing changes because the treasury is covering central bank's losses and therefore central bank's net worth is kept at the initial value.

In the next section, I am going to analyze the case in which the central bank retains financial independence by refusing any treasury's support beyond the initial capitalization. I am going to show that by purchasing risky securities it can lose control of the price level.

\subsection{Results without treasury's support}

I still maintain the assumption that at time $t_{0}$ the treasury provides the initial capital through which the central bank starts its operations. However, after time $t_{0}$, remittances are assumed to be non-negative, $T_{t}^{C} \geq 0$, excluding any possible support from the treasury. In particular, I assume that the central bank transfers all its income to the treasury provided nominal net worth is not below the initial level $\bar{N} \cdot{ }^{3}$ But, as nominal net worth falls below $\bar{N}$ because of negative profits, the central bank rebuilds it by retaining earnings up to the point in which the initial level $\bar{N}$ is recovered. Therefore for each $t>t_{0} T_{t}^{C}=\max \left(\Psi_{t}^{C}, 0\right)$ whenever $N_{t-1}^{C} \geq \bar{N}$ and $T_{t}^{C}=0$ if $N_{t-1}^{C}<\bar{N}$. This remittances' policy has a real-world counterpart in the deferred-asset regime currently used by the Federal Reserve System for which, whenever capital falls, the central bank stops making remittances and accounts for a deferred asset in its balance sheet paid later by retained earnings. Only once the deferred asset is paid in full, the central bank returns to rebate profits to the treasury.

I now define the two monetary/fiscal policy regimes which are the closest counterparts to those of Definitions ?? and ??, with the appropriate qualifications. The following will be used for deflationary spirals.

Definition 4 Define the following monetary/fiscal policy regime setting the sequences $\left\{i_{t}, T_{t}^{F}, T_{t}^{C}, X_{t}, D_{t}^{C}\right\}_{t=t_{0}}^{\infty}$ : i) the policy rule (??), with $\phi>0$, specifies $i_{t}$, ii) $T_{t}^{F}=-T_{t}^{C}$ at each date $t \geq t_{0}$, iii) $T_{t_{0}}^{C}=-P_{t_{0}} n_{t_{0}}^{C}$ for some $n_{t_{0}}^{C}>0, T_{t}^{C}=\max \left(\Psi_{t}^{C}, 0\right)$ if $N_{t-1}^{C} \geq \bar{N}$ and $T_{t}^{C}=0$ if $N_{t-1}^{C}<\bar{N}$ for $t>t_{0}$; iv) $X_{t}>0$ and v) $D_{t}^{C}>0$ for each $t \geq t_{0}$.

\footnotetext{
${ }^{3}$ I define $\bar{N} \equiv P_{t_{0}} n_{t_{0}}^{C}$.
} 
The only difference with respect to Definition ?? is in the specification iii) which excludes transfers from the treasury to the central bank. The following monetary/fiscal policy regime instead replaces that of Definition ?? to trim inflationary spirals.

Definition 5 Define the following monetary/fiscal policy regime setting the sequences $\left.\left\{i_{t}, T_{t}^{F}, T_{t}^{C}, X_{t}, D_{t}^{C}\right\}_{t=t_{0}}^{\infty}: i\right)$ the policy rule (??), with $\phi>0$, specifies $i_{t}$, ii) $T_{t}^{F}=-T_{t}^{C}$ at each date $t \geq t_{0}$, iii) $T_{t_{0}}^{C}=-P_{t_{0}} n_{t_{0}}^{C}$ for some $n_{t_{0}}^{C}>0$ and $T_{t}^{C}=\max \left(\Psi_{t}^{C}, 0\right)$ if $N_{t-1}^{C} \geq \bar{N}$ and $T_{t}^{C}=0$ if $N_{t-1}^{C}<\bar{N}$ and for

$t_{0}<t<\tilde{t}$ while $\frac{T_{t}^{C}}{P_{t}}=\frac{1-\beta}{\beta} \frac{P_{t_{0}}}{P^{*}} n_{t_{0}}^{C}$ for each $t \geq \tilde{t}$ and for some $\tilde{t}>t_{0}$ provided $N_{\tilde{t}-1}^{C} \geq \bar{N}$; iv) $X_{t}>0$ and $\left.v\right) D_{t}^{C}>0$ for each $t \geq t_{0}$.

In Definition 5 one important aspect to underline is that the switch to a real remittances rule (see item iii) is triggered at a generic time $\tilde{t}>t_{0}$ only if the previous-period net worth is at the initial threshold $\bar{N}$. I am also going to assume that time $\tilde{t}$ can be postponed until this occurs.

\subsubsection{Interest-rate shock}

This Section shows that in the case of an unforeseen interest-rate shock there can be multiple solutions: the price stability equilibrium coexists with inflationary spirals, whereas deflationary solutions are ruled out.

Before presenting Propositions and Proofs, let me discuss some ingredients that will be relevant for the analysis. First, note that using (??) into (12), the price of long-term bonds $Q_{t}$ can be expressed as a function of $P_{t}$, that is $Q_{t}=Q\left(P_{t}\right)$, which has an upper-bound value of $\bar{Q}=1 /(1-\delta)$ and is decreasing in $P_{t} \cdot{ }^{4}$ As a consequence, I can also write the return on long-term securities and central bank's profits at time $t$ as a function of $P_{t}$, that is $r\left(P_{t}\right)$ and $\Psi^{C}\left(P_{t}\right)$ respectively, where profits are given by

$$
\Psi^{C}\left(P_{t}\right)=i_{t-1} N_{t-1}^{C}+\left(r\left(P_{t}\right)-i_{t-1}\right) Q_{t-1} D_{t-1}^{C} .
$$

Given its dependence on $r_{t}$ and therefore on $Q_{t}$, the level reached by central bank's net worth at time $t$ is also a function of $P_{t}$

$$
\begin{aligned}
& N^{C}\left(P_{t}\right)=N_{t-1}^{C}+\Psi^{C}\left(P_{t}\right)-\max \left(\Psi_{t}^{C}\left(P_{t}\right), 0\right) \quad \text { if } \quad N_{t-1}^{C} \geq \bar{N} \\
& N^{C}\left(P_{t}\right)=N_{t-1}^{C}+\Psi^{C}\left(P_{t}\right) \quad \text { if } N_{t-1}^{C}<\bar{N}
\end{aligned}
$$

\footnotetext{
${ }^{4}$ In this subsection, I am assuming that $\varkappa_{t}=0$ at all times.
} 
which follows from (??) where I have used the remittances' rule of Definitions 4 and 5.

First, I show that deflationary paths are ruled out extending Proposition $? ?$

Proposition 6 Given the monetary/fiscal policy regime of Definition 4 it follows that $P_{t} \geq P^{*}$ in equilibrium for each $t \geq t_{0}$.

Proof. First I show that solution paths in which $P_{t}<P^{*}$ at time $t_{0}$ are ruled out. Since the deflationary path is foreseen at time $t_{0}$, profits are always positive and therefore Proposition ?? applies. I now consider the case in which prices are first foreseen to be constant, $P_{t}=P^{*}$, for each time $t \geq t_{0}$ then, unexpectedly, they fall, $P_{\hat{t}}<P^{*}$, at a generic time $\hat{t}$ (with $\hat{t}>t_{0}$ ) and from that time they follow a deflationary path according to (??). When unexpectedly $P_{t}<P^{*}$ at $\hat{t}>t_{0}$, the return on long-term bonds $r\left(P_{\hat{t}}\right)$ exceeds $i_{\hat{t}-1}$, and profits remain positive. Therefore the proof of Proposition ?? still applies.

The intuition for why deflationary solutions are eliminated even when the central bank holds long-term bonds depends on the fact that an unexpected fall in the price level produces a rise in the return on long-term bonds and therefore profits remain positive and net worth constant, given the remittances rule. A constant nominal net worth rules out deflationary solutions as the proof of Proposition ?? shows.

I will now discuss the existence of inflationary equilibria.

Proposition 7 Given the monetary/fiscal policy regime of Definition 5 it follows that there are multiple equilibria. There is an equilibrium in which $P_{t}=P^{*}$ for each $t \geq t_{0}$ and an equilibrium in which $P_{t}=P^{*}$ for each $t_{0} \leq t<\hat{t}$ and unexpectedly $P_{t}>P^{*}$ at time $\hat{t}$, with $t_{0}<\hat{t}<\tilde{t}-1$, and in which prices follow an inflationary spiral afterward according to (??) and net worth is zero for each $t \geq \hat{t}$.

Proof. First, I show that solution paths in which $P_{t}>P^{*}$ at time $t_{0}$ are ruled out. Since the inflationary path is foreseen at time $t_{0}$, profits are always positive and therefore Proposition ?? applies. I will now check for solution paths in which it is first expected that $P_{t}=P^{*}$ for each $t \geq t_{0}$ and then unexpectedly $P_{\hat{t}}>P^{*}$ at $\hat{t}>t_{0}$ with an inflationary path following equation (??) afterward. In this case, $r\left(P_{\hat{t}}\right)<i_{\hat{t}-1}$ and $r\left(P_{t}\right)=i_{t-1}$ at all other times. 
Several cases are possible. First note that Proposition ?? applies if the time $\hat{t}$ is larger or equal to $\tilde{t}-1$, i.e. $\hat{t} \geq \tilde{t}-1$, given that the remittances rule requires that $\frac{T_{t}^{C}}{P_{t}}=\frac{1-\beta}{\beta} \frac{P_{t_{0}}}{P^{*}} n_{t_{0}}^{C}$ for each $t \geq \tilde{t}$. Indeed

$$
\frac{N^{C}\left(P_{\hat{t}}\right)}{P_{\hat{t}}}=\sum_{T=\hat{t}+1}^{\infty} \beta^{T-\hat{t}} \frac{T_{T}^{C}}{P_{T}}=\frac{P_{t_{0}} n_{t_{0}}^{C}}{P^{*}} .
$$

Note that $N^{C}\left(P_{\hat{t}}\right)$ is monotone non-increasing with $P_{\hat{t}}$ and, therefore, the left-hand side of the above equation is decreasing with $P_{\hat{t}}>P^{*}$ and is equal to the right-hand side only when $P_{\hat{t}}=P^{*}$. Therefore paths in which $P_{\hat{t}}>P^{*}$ with $\hat{t} \geq \tilde{t}-1$ are not equilibria. Let us focus on the case in which $P_{\hat{t}}>P^{*}$ at $\hat{t}<\tilde{t}-1$. I distinguish several possibilities. First, if $\Psi^{C}\left(P_{\hat{t}}\right)>0, T_{t}^{C}=\Psi_{t}^{C}$ in all periods and therefore Proposition ?? applies ruling out paths in which $P_{\hat{t}}>P^{*}$. Second, if $\Psi^{C}\left(P_{\hat{t}}\right)<0$ and $N^{C}\left(P_{\hat{t}}\right)>0$, then net worth is increasing after time $\hat{t}$ because profits are going to be positive since the inflationary path is perfectly foreseen after $\hat{t}$. Therefore, net worth increases and it will reach the value $\bar{N}^{C}$ at time $\tilde{t}-1$ since indeed I have assumed that time $\tilde{t}$ can be postponed until that occurs. Therefore at that time, the following equivalence holds

$$
\frac{\bar{N}^{C}}{P_{\tilde{t}-1}}=\sum_{T=\tilde{t}}^{\infty} \beta^{T+1-\hat{t}} \frac{T_{T}^{C}}{P_{T}}=\frac{P_{t_{0}} n_{t_{0}}^{C}}{P^{*}}
$$

which can only be satisfied if and only if $P_{\tilde{t}-1}=P^{*}$ ruling out path in which $P_{\tilde{t}-1}>P^{*}$ and therefore $P_{\hat{t}}>P^{*}$ at $\hat{t}<\tilde{t}-1$. Third, consider the case in which $\Psi^{C}\left(P_{\hat{t}}\right)<0$ and also net worth is negative $N^{C}\left(P_{\hat{t}}\right)<0$ then net worth remains negative in all the following periods since profits are negative. Net worth will follow the path $N_{t}^{C}=\left(1+i_{t-1}\right) N_{t-1}^{C}$ since remittances will be always zero and the real remittances policy is not triggered. Given that $1+i_{t}=\beta^{-1} P_{t+1} / P_{t}$, real net worth will decrease at a rate $N_{t} / P_{t}=\beta^{-1} N_{t-1} / P_{t-1}<0$ violating the transversality condition. Therefore, these inflationary paths are not equilibria. Finally consider the case in which $\Psi^{C}\left(P_{\hat{t}}\right)<0$ and net worth is zero, $N^{C}\left(P_{\hat{t}}\right)=0$. Then net worth remains zero since profits are zero for each $t>\hat{t}$. Remittances are also going to be zero given the remittances' policy. This is an equilibrium since it will not violate the transversality condition.

Finally note that the results of this section are related to Del Negro and Sims (2015) but with an important difference. In their analysis, multiplicity 
appears as a shift to a different interest-rate rule, since it is $P^{*}$, the inflation target in their case, that changes across equilibria. In my analysis, the policy rule remains unchanged and the multiplicity arises along the multiple solutions that (??) implies.

\subsubsection{Credit shock}

I consider now the consequences of an unexpected realization of a credit event showing that the stationary solution $P_{t}=P^{*}$ stops to be an equilibrium when the credit event is sizeable whereas divergent solutions (inflationary and deflationary) might also emerge as equilibria.

Starting from a perfect foresight equilibrium in which $\varkappa_{t}=0$ at all times, assume that at a generic time $\hat{t}>t_{0}$ long-term securities are unexpectedly seized, even partially, at the rate $0<\varkappa \leq 1$. The time- $\hat{t}$ return on long-term bonds unexpectedly falls which could lead to negative profits and to a fall in net worth.

The profit function at time $\hat{t}$ is now also function of $\varkappa$

$$
\Psi^{C}\left(\varkappa, P_{\hat{t}}\right)=i_{\hat{t}-1}\left(N_{\hat{t}-1}^{C}+M_{\hat{t}-1}^{C}\right)+\left(r\left(\varkappa, P_{\hat{t}}\right)-i_{\hat{t}-1}\right) Q_{\hat{t}-1} D_{\hat{t}-1}^{C}
$$

given the dependence of the return function $r\left(\varkappa, P_{\hat{t}}\right)$ on $\varkappa$. Central bank's net worth at time $\hat{t}$ is given by

$$
\begin{aligned}
& N^{C}\left(\varkappa, P_{\hat{t}}\right)=N_{\hat{t}-1}^{C}+\Psi^{C}\left(\varkappa, P_{\hat{t}}\right)-\max \left(\Psi^{C}\left(\varkappa, P_{\hat{t}}\right), 0\right) \quad \text { if } \quad N_{\hat{t}-1}^{C} \geq \bar{N}, \\
& N^{C}\left(\varkappa, P_{\hat{t}}\right)=N_{\hat{t}-1}^{C}+\Psi^{C}\left(\varkappa, P_{\hat{t}}\right) \quad \text { if } \quad N_{\hat{t}-1}^{C}<\bar{N} \text {. }
\end{aligned}
$$

In what follows, I restrict the analysis to equilibria in which the price level can jump in an unexpected way at the time in which the credit shock hits. The following Proposition describes the equilibria in this case.

Proposition 8 Consider an equilibrium in which $\varkappa_{t}=0$ at each date and unexpectedly $\varkappa_{t}=\varkappa$ at time $\hat{t}>t_{0}$. Given the monetary/fiscal policy regime of Definition 4 it follows that: i) $P_{t}=P^{*}$ in equilibrium for each $t \geq t_{0}$ if and only if $N^{C}\left(\varkappa, P^{*}\right)=N_{\hat{t}-1}^{C}+\Psi^{C}\left(\varkappa, P^{*}\right) \geq 0$ at time $\hat{t}$ and ii) there is an equilibrium in which $P_{t}=P^{*}$ for each $t_{0} \leq t<\hat{t}$ and unexpectedly $P_{\hat{t}}<P^{*}$ with a deflationary path afterward which follows (??) if and only if $N^{C}\left(\varkappa, P^{*}\right)=N_{\hat{t}-1}^{C}+\Psi^{C}\left(\varkappa, P^{*}\right)<0$ and $N^{C}\left(\varkappa, P_{\hat{t}}\right)=0$ at time $\hat{t}$. 
Proof. There are different cases to analyze. If profits are still positive, following the $\varkappa$-shock evaluated at the target price $P^{*}$, i.e. $\Psi^{C}\left(\varkappa, P^{*}\right)>$ 0 , then it is the case that $\Psi^{C}\left(\varkappa, P_{\hat{t}}\right)>\Psi^{C}\left(\varkappa, P^{*}\right)$ for any unexpected fall in the price level at time $\hat{t}>t_{0}, P_{\hat{t}}<P^{*}$, and therefore $T_{t}^{C}=\Psi_{t}^{C}>$ 0 for each $t \geq \hat{t}$ implying $N_{t}^{C}=\bar{N}$ for each $t \geq \hat{t}$. This result implies that deflationary paths are excluded as shown in Proposition ??. Consider now the case in which $\Psi^{C}\left(\varkappa, P^{*}\right)<0$ at time $\hat{t}$ but $N^{C}\left(\varkappa, P^{*}\right)=N_{\hat{t}-1}^{C}+$ $\Psi^{C}\left(\varkappa, P^{*}\right)>0$. Therefore $N^{C}\left(\varkappa, P_{\hat{t}}\right)>N^{C}\left(\varkappa, P^{*}\right)$ for any unexpected fall in the price level at time $\hat{t}, P_{\hat{t}}<P^{*}$. Given that net worth is positive, profits will be positive (in a perfect-foresight equilibrium) after time $\hat{t}$ and they reach the threshold $\bar{N}^{C}$ in a finite period of time. After that period, net worth will be constant at the level $\bar{N}^{C}$. Therefore Proposition ?? applies and deflationary paths are rule out. Consider now the case in which $\Psi^{C}\left(\varkappa, P^{*}\right)<$ 0 at time $\hat{t}$ and $N^{C}\left(\varkappa, P^{*}\right)=N_{\hat{t}-1}^{C}+\Psi^{C}\left(\varkappa, P^{*}\right)=0$. Given that net worth is zero, profits are zero after time $\hat{t}$ and therefore also remittances. Net worth remains zero without violating the transversality condition. $P_{t}=$ $P^{*}$ is an equilibrium at all times. On the contrary, paths in which $P_{t}=$ $P^{*}$ for each $t_{0} \leq t<\hat{t}$ and $P_{\hat{t}}<P^{*}$ at time $\hat{t}$ with deflationary paths in the following periods according to (??) are not equilibria since they lead to violation of the transversality condition. In the case in which $N^{C}\left(\varkappa, P^{*}\right)=$ $N_{\hat{t}-1}^{C}+\Psi^{C}\left(\varkappa, P^{*}\right)<0$ path in which $P_{t}=P^{*}$ at each $t$ cannot be equilibria since net worth is negative, profits are negative and net worth diverges to negative values at a rate violating the transversality condition, i.e. $N_{t} / P_{t}=$ $\beta^{-1} N_{t-1} / P_{t-1}<0$ for each $t \geq \hat{t}$. However, if prices fall at time $\hat{t}$ when the credit shock hits such that $N^{C}\left(\varkappa, P_{\hat{t}}\right)=0$ then profits will be zero afterward. Zero remittances implies that net worth remains constant at zero without violating the transversality condition. Therefore there can be equilibria with a deflationary path for a sizeable credit shock.

The above Proposition shows that depending on the size of the credit shock there can be different types of equilibria. If the credit shock is small, price stability is an equilibrium. On the contrary, for large credit shock, deflationary equilibria arise and they are such that $N^{C}\left(\varkappa, P_{\hat{t}}\right)=0$ at time $\hat{t}$ for some $P_{\hat{t}}$, with $P_{\hat{t}}<P^{*}$, and moreover net worth remains zero in all the following periods. The next Proposition shows instead that whenever the size of the shock is small, there are multiple equilibria. If $N^{C}\left(\varkappa, P^{*}\right)>0$, the price stability equilibrium coexists with an inflationary equilibrium such that $N^{C}\left(\varkappa, P_{\hat{t}}\right)=0$ at time $\hat{t}$ for some $P_{\hat{t}}$, with $P_{\hat{t}}>P^{*}$. 
Proposition 9 Consider an equilibrium in which $\varkappa_{t}=0$ at each date and unexpectedly $\varkappa_{t}=\varkappa$ at time $\hat{t}>t_{0}$. Given the monetary/fiscal policy regime of Definition 5 , it follows that: i) $P_{t}=P^{*}$ in equilibrium for each $t \geq t_{0}$ if and only if $N^{C}\left(\varkappa, P^{*}\right)=N_{\hat{t}-1}^{C}+\Psi^{C}\left(\varkappa, P^{*}\right) \geq 0$ at time $\hat{t}$ and $\left.i i\right)$ whenever $N^{C}\left(\varkappa, P^{*}\right)>0$ there is also an equilibrium in which $P_{t}=P^{*}$ for each $t_{0} \leq$ $t<\hat{t}$, with $\hat{t}<\tilde{t}-1$, and unexpectedly $P_{\hat{t}}>P^{*}$ with an inflationary path which follows (??) if and only if $N^{C}\left(\varkappa, P_{\hat{t}}\right)=0$.

Proof. I will now check whether paths in which $P_{t}=P^{*}$ for each $t \geq t_{0}$ and prices unexpectedly jump at the time of the credit shock and follow an inflationary path in the following periods, i.e. $P_{t}>P^{*}$ for each $t \geq \hat{t}>t_{0}$ according to (??), are equilibria. Recall that the remittances rule requires that $\frac{T_{t}^{C}}{P_{t}}=\frac{1-\beta}{\beta} \frac{P_{t_{0}}}{P^{*}} n_{t_{0}}^{C}$ for each $t \geq \tilde{t}$ at time $\tilde{t}$ such that $N_{\tilde{t}-1}^{C}=\bar{N}^{C}$. Since $N^{C}\left(\varkappa, P^{*}\right)<\bar{N}^{C}$ and $N^{C}\left(\varkappa, P_{\hat{t}}\right)<\bar{N}^{C}$ for any unexpected increase of prices, i.e. $P_{\hat{t}}>P^{*}$, time $\tilde{t}-1$ can only occur after time $\hat{t}$. Consider then the case in which $\hat{t}<\tilde{t}-1$. If $N^{C}\left(\varkappa, P_{\hat{t}}\right)>0$ for some $P_{\hat{t}}>P^{*}$ then profits are positive after time $\hat{t}$ and net worth increases. Therefore net worth can reach the threshold $\bar{N}^{C}$ at a future time $\tilde{t}-1$. The intertemporal budget constraint at time $\tilde{t}-1$ is given by

$$
\frac{\bar{N}^{C}}{P_{\tilde{t}-1}}=\sum_{T=\tilde{t}}^{\infty} \beta^{T+1-\tilde{t}} \frac{T_{T}^{C}}{P_{T}}=\frac{P_{t_{0}} n_{t_{0}}^{C}}{P^{*}}
$$

which can only be satisfied if and only if $P_{\tilde{t}-1}=P^{*}$ ruling out paths in which $P_{\tilde{t}-1}>P^{*}$ and therefore $P_{\hat{t}}>P^{*}$ at $\hat{t}<\tilde{t}-1$. If instead $N^{C}\left(\varkappa, P^{*}\right)>$ $N^{C}\left(\varkappa, P_{\hat{t}}\right)=0$, profits are zero, remittances are zero and net worth remains zero in all the following periods. The inflationary solution in which prices jump at $\hat{t}$, with $P_{\hat{t}}>P^{*}$, and follow an inflationary path consistently with (??) is an equilibrium. Finally when $N^{C}\left(\varkappa, P_{\hat{t}}\right)<0$, net worth follows the path $N_{t}^{C}=\left(1+i_{t-1}\right) N_{t-1}^{C}$ and given that $1+i_{t}=\beta^{-1} P_{t+1} / P_{t}$, real net worth will decrease at a rate $N_{t} / P_{t}=\beta^{-1} N_{t-1} / P_{t-1}<0$ violating the transversality condition. As shown in Proposition 8, whenever $N^{C}\left(\varkappa, P^{*}\right) \geq 0$ price stability, i.e. $P_{t}=P^{*}$ at each date $t$, is an equilibrium. 